\title{
A probabilistic approach to heterogeneity in space plasmas: the case of magnetic field intensity in solar wind
}

\author{
G. Consolini ${ }^{1}$, B. Bavassano ${ }^{2}$, and P. De Michelis ${ }^{2}$ \\ ${ }^{1}$ INAF-Istituto di Fisica dello Spazio Interplanetario, 00133 Roma, Italy \\ ${ }^{2}$ Istituto Nazionale di Geofisica e Vulcanologia, 00143 Roma, Italy
}

Received: 17 November 2008 - Revised: 6 March 2009 - Accepted: 18 March 2009 - Published: 7 April 2009

\begin{abstract}
Since the early 90s it was shown that Probability Distribution Functions (PDFs) of small scale differences (fluctuations) of several quantities in space plasmas display significant departures from Gaussianity. The non-Gaussian shape of PDFs was ascribed to intermittency and discussed in the framework of intermittent MHD turbulence. Here, we put the attention to the PDF of magnetic field intensity instead of its differences showing how the PDF of such quantity in a quiet solar wind can be related with the occurrence of heterogeneity. In detail, we derive the shape of the PDFs by simple statistical considerations based on the concept of subordination in probability theory. An interpretation and a discussion in terms of existing coherent magnetic structures in a mechanical near-equilibrium state are also presented.
\end{abstract}

\section{Introduction}

In the last 20 years we experienced changes in the approach to the study of solar-system space plasmas. Several novel concepts dealing with the evolution of nonlinear, turbulent and complex systems have been introduced to explain the dynamics of space plasmas on both global and small scales. The principal merit of such novel approaches has been to underline the relevance of stochastic fluctuations and coherent structures in the dynamics of solar-system plasmas (see Tetrault, 1992a, 1992b; Chang, 1992, 1999; Klimas et al., 1996; Chang et al., 2004; Zelenyi and Milovanov, 2004; Bruno and Carbone, 2005).

One of the most peculiar features of magnetospheric and interplanetary space plasmas is the non-Gaussian and leptokurtic shape of the Probability Distribution Functions (PDFs) of the small scale fluctuations of several quantities,

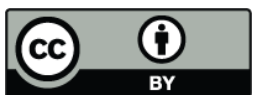

Correspondence to: G. Consolini (giuseppe.consolini@ifsi-roma.inaf.it) where the term "fluctuation" generally refers to temporal and/or spatial differences of the observed quantities. For example, since the first half of $90 \mathrm{~s}$ (Marsch and Tu, 1994) it is well-known that magnetic field and velocity fluctuations in the solar-wind are characterized by non-Gaussian PDFs at small-scales, approaching to Gaussian distributions at larger scales. Similar non-Gaussian distibutions have been detected in the magnetospheric regions (see for example Angelopoulos et al., 1999; Weygand et al., 2005; Vörös et al., 2005). This feature is generally ascribed to the occurrence of intermittency in a turbulent medium (Frisch, 1995) and discussed in the framework of MHD turbulence (see Bruno and Carbone, 2005). As a matter of fact, intermittency phenomenon, which, nowadays, is a central issue in hydrodynamic and magnetohydrodynamic turbulence studies, manifests in a statistics dominated by very large fluctuations, sparsely distributed in space. From a physical point of view, intermittency refers to the existence of anomalous dimensions in the sense of critical phenomena and/or quantum field theory that emerges from the growing of the fluctuations in the course of the cascading process. As a result intermittency manifests in a nonuniformity of the spatial distribution of dissipative structures in turbulent systems, in a departure from Gaussian statistics in the course of the cascading process and may be described in terms of a multifractal cascading process (see Parisi and Frisch, 1985; Frisch, 1995; Biskamp, 1993; Sorriso-Valvo et al., 2001).

Due to the ubiquitousness of non-Gaussian distributions in many physical scenarios several models have been proposed. This is particularly true in the framework of hydrodynamic and magnetohydrodynamic turbulence, where different approaches have been proposed to understand the leptokurtic nature of small-scale PDFs (Castaing et al., 1990; Frisch and Sornette, 1997; Beck and Cohen, 2003; Leubner and Vörös, 2005a, b). These different approaches move from different physical and statistical assumptions: the composition law (Castaing et al., 1990; Beck and Cohen, 2003;

Published by Copernicus Publications on behalf of the European Geosciences Union and the American Geophysical Union. 
Jung and Swinney, 2005), the Tsallis' statistics (Leubner and Vörös, 2005a, b) and the Extreme Deviation Theory (Frisch and Sornette, 1997). For example, the widely used model (Castaing et al., 1990) is derived from the assumption that the PDF of the velocity flucluations $\delta v_{r}$ at the separation scale $r$ can be written as a convolution of a Gaussian distribution $P_{G}\left(\delta v_{r} \mid \sigma\right)$ with a weight function $G_{\lambda}(\sigma)$ representing the statistical weight of the Gaussian PDF characterized by the standard deviation $\sigma$, i.e.

$P_{\lambda}\left(\delta v_{r}\right)=\int G_{\lambda}(\sigma) P\left(\delta v_{r} \mid \sigma\right) d \sigma$

where $\lambda$ is a parameter related to the width of $G_{\lambda}(\sigma)$. A similar approach is used in the case of superstatistics (Beck and Cohen, 2003).

Although the departure from Gaussian distributions in space plasmas is generally interpreted as the signature of intermittent MHD turbulence, since the mid-90s it was proposed that the existence of coherent magnetic and/or plasma structures might play a relevant role. For example, Tu and Marsch (1993, 1995) proposed that the understanding of some observed features of solar wind required a scenario involving a mixture of stochastic fluctuations and coherent advected structures. More recently, Chang et al. (2004) have shown that large-amplitude fluctuations, responsible for non-Gaussian statistics, are related to the existence of coarse-grained multiscale coherent structures (Chang, 1999), emerging from the cross-scale coupling of stochastic fluctuations, as it has also been validated by numerical 2DMHD simulations (Wu and Chang, 2000; Chang et al., 2004). In several space-plasma scenarios (solar-wind, Earth's magnetotail plasma sheet, etc.) such coherent structures can take the shape of flux tubes, recalling the interpretation of the interplanetary observations given by several authors (Ness et al., 1966; McCracken and Ness, 1966; Mariani et al., 1973; Tu and Marsch, 1990, 1993; Bruno et al., 2001; Borovsky, 2008). Furthermore, the existence of coarse-grained multiscale coherent structures necessarily involves the emergence of long-range correlations. Indeed, the appearance of coherent structures in a physical system is equivalent to the appearance of a certain degree of ordering, and thus according to Nicolis and Prigogine (1977) we can say that we are in presence of a phenomenon of "ordering through correlations". Recently Milovanov and Zelenyi (2000), discussing the relationship between "coarse-grained" systems and "kappa" distribution functions, have argued that the ordering through correlations can involve a "coarse-grained" topology of the system, and that this may be related with the emergence of a non-Gaussian statistics. From the aforementioned points it turns out that "coarse-grained" stochasticity may play a relevant role in space plasmas.

This paper is intended to focus the attention on the statistics of the magnetic field intensity at short time-scale and to discuss the observed departure from Gaussian/Maxwellian distribution function in terms of "coarse-grained" magnetic structures. In detail, we will show how the statistics of magnetic field intensity is in agreement with a simple probabilistic model based on subordination method (Feller, 1971) and involving rare event statistics.

The paper is organized as follows: Sect. 2 introduces a probabilistic model for heterogeneous systems and some basic concepts dealing with statistical subordination; Sect. 3 shows a comparison between the proposed model and observations of interplanetary magnetic field intensity in a longstanding slow solar-wind period; in Sect. 4 we trace out our conclusions.

\section{Heterogeneity and rare event statistics}

As well documented by the existing literature (see previous Section) one of the most interesting features of the space plasma media is the small scale heterogeneity of several quantities. A possible way to approach the observed heterogeneity is to model the statistical features of some quantities by randomizing the associated control parameters. Then following the subordination/directing process (Feller, 1971; Lavenda, 1995a), a novel distribution function for the observables and its small scale increments can be introduced. Moving from a n-dimensional Maxwell distribution the subordination/directing method has been already applied by one of us (Consolini, 2007) to attempt a description of the nonGaussian statistics of the small-scale temporal differences of magnetic field fluctuations as observed in the magnetotail regions. In what follows, we apply the above technique to rare event distribution functions, getting different shapes of the subordinate distributions. Anyway, we start by introducing the method (see Feller, 1971; Lavenda, 1995a).

Let us start by considering a random variable $x$ relative to a stochastic Markov process (the reader may think about a Brownian diffusing particle), and distributed according to a Gaussian distribution:

$\varphi\left(x ; s_{0}\right)=\frac{1}{\sqrt{2 \pi s_{0}}} e^{-x^{2} / 2 s_{0}}$,

where $s_{0}$ is a parameter. The probability distribution (2) can be thought as a conditional probability, $\varphi\left(x ; s_{0}\right) \equiv \varphi\left(x \mid s_{0}\right)$, where the parameter $s_{0}$ plays the role of a condition. Starting from the above distribution for the $x$ variate we look for the distribution of the parameter $s$ at which the stochastic process attains a value $x_{0}$ of the variate. That can be obtained by applying the following transform introduced by Lévy, (see e.g. Lavenda, 1995a)

$x=x_{0} \sqrt{\frac{s_{0}}{s}}$

where $s_{0}$ and $x_{0}$ are two characteristic values. Applying the Lévy transform (3), the Gaussian distribution (2) is converted 


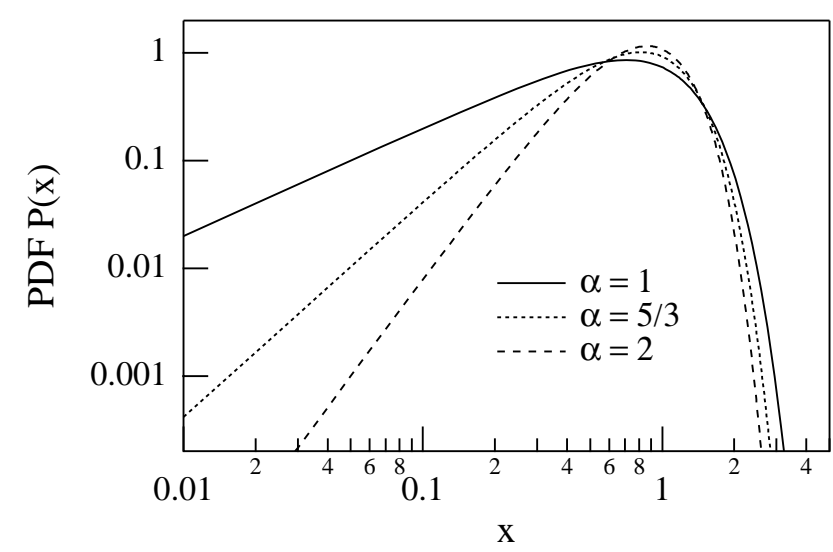

Fig. 1. The generalized Weibull distribution for $\theta=1, \beta=2$ and different values of $\alpha$.

into the strictly stable Lévy distribution with characteristic exponent $\alpha=1 / 2$, i.e.

$\varphi\left(s \mid x_{0}\right)=\frac{x_{0}}{s} \sqrt{\frac{1}{2 \pi s}} e^{-x_{0}^{2} / 2 s}$.

Consequently, the application of the Lévy transform is equivalent to a randomization procedure of the parameter $s$.

Moving from the distribution for the parameter $s$ it is possible to construct a novel Markov process by applying the directing process (Feller, 1971), characterized by the density distribution (4). Thus, for the novel Markov process we can write the following expression

$\varphi\left(x \mid x_{0}\right)=\int_{0}^{\infty} \varphi(x \mid s) \varphi\left(s \mid x_{0}\right) d s$

where it has been assumed $s=s_{0}$. For $x$ variate, described above, one obtains

$\varphi\left(x \mid x_{0}\right)=\frac{1}{\pi} \frac{x_{0}}{x^{2}+x_{0}^{2}}$

which is the well known Cauchy distribution, and where the $x_{0}$ parameter plays the role of a characteristic value for the $x$ variate.

As a result of this procedure, it follows that the Cauchy density distribution is subordinated to the Gaussian one by randomizing the parameter $s_{0}$. This is exactly the meaning of subordination in probability theory. Furthermore, as clearly shown in Lavenda's book (1995a) the subordination method can be successfully applied to derive the probability distribution of one component of a composite system (see also Lavenda, 1995b).

Actually, in many physical systems the distribution functions of several physical quantities do not follow a simple Gaussian (Maxwellian) distribution, showing for instance power-law tails. This is particularly true in the case of complex systems and for global observables in correlated sys- tems. For such classes of physical systems the distribution functions of the fluctuations of observables are generally characterized by an asymmetric shape, with an exponential/power law tail on one side and a rapid fall-off on the other side (Chapman et al., 2002; Sornette, 2003; Bertin and Clusel, 2006). One way to model the observed distributions is to considered the class of distributions for extreme values (Sornette, 2003; Gumbel, 2004), such as the so-called $e x$ treme value generalized distributions, introduced by Bertin and Clusel (2006) to model the sum of correlated variables. In particular, we start from the case of generalized Weibull distribution (Bertin and Clusel, 2006), which is valid in the case of sum of correlated variables characterized by an upper bound:

$$
\varphi(x \mid \theta, \alpha, \beta)=\beta \frac{\alpha^{\alpha}}{\Gamma(\alpha)} \frac{x^{\alpha \beta-1}}{\theta^{\alpha \beta}} \exp \left(-\alpha\left(\frac{x}{\theta}\right)^{\beta}\right)
$$

where $x \in[0, \infty), \alpha$ and $\beta \in \mathcal{R}^{+}$, and $\theta$ is a scale parameter. The parameter $\alpha$ quantifies the degree of correlation (i.e. the deviation from Central Limit Theorem) in the sum of the random variables generating the limiting distribution $\varphi(x \mid \theta, \alpha, \beta)$, and $\alpha \rightarrow 1$ for highly correlated systems/signals (Bertin and Clusel, 2006). Figure 1 shows an example of this distribution for $\theta=1, \beta=2$ and different $\alpha$ values.

Considering the generalized Weibull distribution (see Eq. 7) and applying the above described subordination/directing process we can construct a novel Markov process characterized by a probability density function (PDF) which assumes the following functional form:

$\Pi\left(x \mid x_{0}, \alpha, \beta\right)=\beta \frac{\Gamma(2 \alpha)}{\Gamma^{2}(\alpha)} \frac{x_{0}^{\alpha \beta} x^{\alpha \beta-1}}{\left(x_{0}^{\beta}+x^{\beta}\right)^{2 \alpha}}$.

This new probability density function is characterized by an asymmetric shape with power-law tails decaying as $x^{\alpha \beta-1}$ $\left(x^{-\alpha \beta-1}\right)$ on left- (right-) hand tail. Figure 2 shows a comparison between the generalized Weibull distribution $\varphi(x \mid$ $\theta, \alpha, \beta)$ and the one $\Pi\left(x \mid x_{0}, \alpha, \beta\right)$ obtained by randomizing the $\theta$ parameter for $(\alpha, \beta)=(1,2)$.

The novel PDF, which we call the randomized Weibull for convenience, is characterized by the following first two moments:

$$
\begin{aligned}
\langle x\rangle & =x_{0} \frac{\Gamma\left(\alpha-\frac{1}{\beta}\right) \Gamma\left(\alpha+\frac{1}{\beta}\right)}{\Gamma^{2}(\alpha)}, \\
\left\langle x^{2}\right\rangle & =x_{0}^{2} \frac{\Gamma\left(\alpha-\frac{2}{\beta}\right) \Gamma\left(\alpha+\frac{2}{\beta}\right)}{\Gamma^{2}(\alpha)},
\end{aligned}
$$

where $\alpha \beta>2$. In general, the existence/convergence of the $n t h$-moment requires that $\alpha \beta>n$ and, thus, for the PDFs of actual physical quantities we should expect the existence of a (exponential) cut-off on the right-hand tail. 


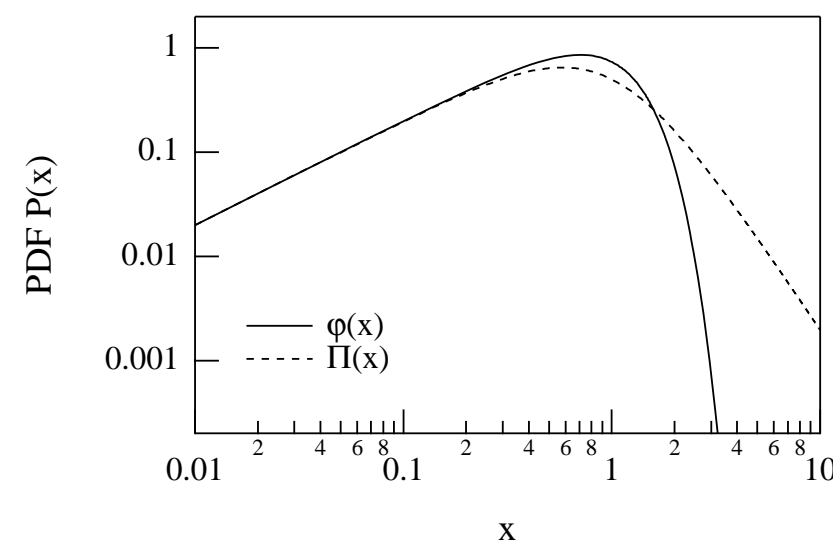

Fig. 2. A comparison of the generalized Weibull distribution $\varphi(x \mid$ $\theta, 1,2)$ (with $\theta=1)$ and the one $\Pi\left(x \mid x_{0}, 1,2\right)$ (with $x_{0}=1$ ) obtained randomizing the parameter $\theta$.

In the case of a simple Markov process (thus with no memory effect at all) it is possible to evaluate the distribution function of the difference $\left(\delta x=x_{i+1}-x_{i}\right)$ between two successive realization of the stochastic process $\left\{x_{i}\right\}$. This can be done by applying the usual probability composition law:

$\psi(\delta x)=\int_{0}^{\infty} \Pi_{\alpha, \beta}\left(x ; x_{0}\right) \Pi_{\alpha, \beta}\left(x+|\delta x| ; x_{0}\right) d x$.

Figure 3 shows an example of the PDF $\psi(\delta x)$ of the differences for $x_{0}=1, \beta=2$ and different $\alpha$ values, as obtained by numerical integration. One very interesting feature of such PDFs is that their shape is leptokurtotic with power-law tails. It is necessary to remark that in the case of Markov process, whose random variables (variates) are distributed according to the generalized Weibull, the PDF of the differences is expected to be Gaussian. Furthermore, to verify Eq. (11) in getting the right PDF of the variate differences $\delta x$, we have simulated a series of $x_{i}$ values distributed according to Eq. (8) with $x_{0}=1, \alpha=1$ and $\beta=2$, and successively compared the PDF of the differences $\delta x$ between two successive random variables and the prediction of Eq. (11). In Fig. 4 we report the comparison. The agreement is excellent.

Before moving to the next Section it is worth noting that in the case of thermodynamic systems the directing process (see Eq. 5) is equivalent to fix an equilibrium condition among the different components of the system itself (Lavenda, 1995a, b).

\section{Model test}

\subsection{Data description}

To test functional expressions derived in the previous Section, we investigate the PDFs of the fluctuations of the magnetic field intensity at short time scale in the solar wind

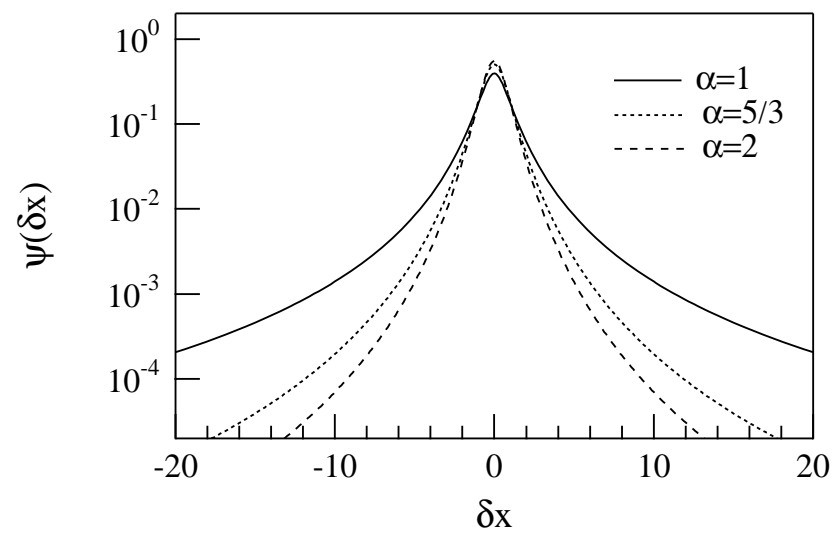

Fig. 3. The PDF $\psi(\delta x)$ of the differences $\delta x$ as evaluated by numerical integration using Eq. (11) for the three cases reported in Fig. 2.

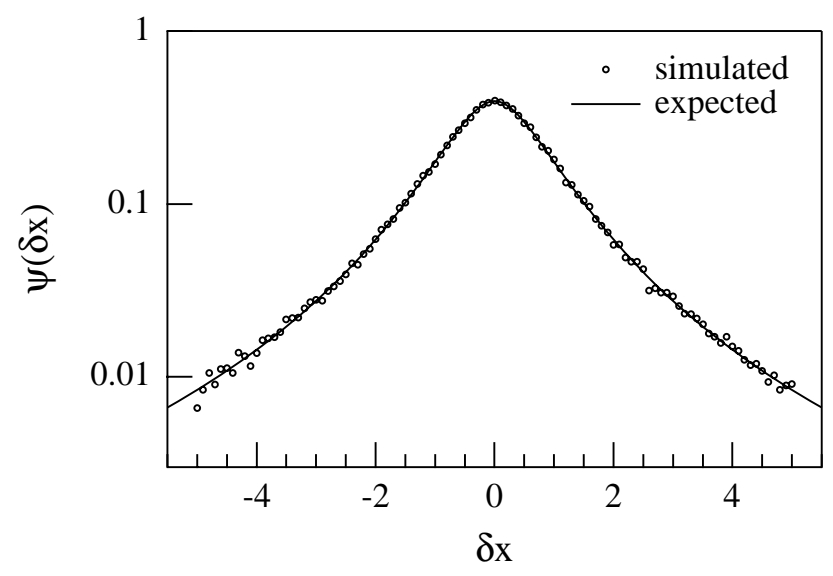

Fig. 4. A comparison between the PDF of a simulated Markov process with variates distributed according to Eq. (8) with $x_{0}=1, \alpha=1$ and $\beta=2$ (white circles) and the expected PDF $\psi(\delta x)$ of the differences $\delta x$ as evaluated by numerical integration using Eq. (11) (solid line).

as observed by Ulysses mission in a period of six months (from 1 October 1997 to 31 March 1998) when Ulysses, near the heliospheric equatorial plane and at about $5 \mathrm{AU}$ $(d=[5.35 \pm 0.06] \mathrm{AU})$, was sampling a very quiet solar wind. The very small variation of the satellite distance during the selected period ensures the radial effect on $B$ to be negligible.

As it will be shown below, this period is also characterized by a strongly reduced content of Alfvénic fluctuations, offering an excellent opportunity to investigate the PDF in a regime of quasi-stationarity with a particular emphasis to the role of structures. Moreover, this period is characterized by a very low and nearly constant radial velocity $\left(v_{R}=[370 \pm 20] \mathrm{km} / \mathrm{s}\right)$ where the associated gradients are extremely small, i.e. it is an extremely quiet period. The plasma $\beta$ is about 1 . 


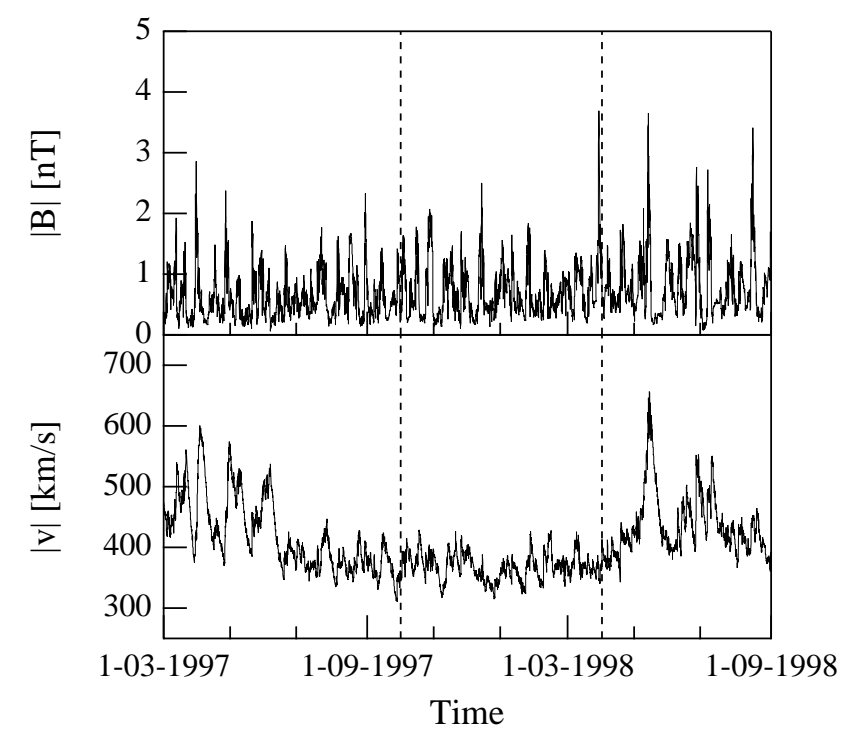

Fig. 5. The magnetic field intensity (upper panel) and the solarwind radial velocity (lower panel), as observed by Ulysses for the period from 01-03-1997 to 01-09-1998. The two vertical dashed lines indicate the time interval here considered.

In detail, we considered $24 \mathrm{~s}$ magnetic field measurements (obtained by averaging $1 \div 2 \mathrm{~s}$ data) and $4 \div 8$ min plasma velocity measurements. Data come from the NASA-CDAWeb service of the NSSDC Data Center. We show the $1 \mathrm{~h}$ magnetic field intensity and plasma radial velocity measurements for the selected period in Fig. 5.

As mentioned before this period is very interesting because the state of the solar wind is mainly characterized by fluctuations with a low correlation of Alfvénic type $(\langle\mathbf{v} \cdot \mathbf{b}\rangle)$ and with a predominance of magnetic energy $\left(E_{b}>E_{v}\right)$. This point is evidenced by the distribution of the two parameters characterizing the solar wind MHD turbulence (see Bavassano et al., 1998, 2000, 2005):

1) the normalized cross-helicity $\sigma_{C}$

$\sigma_{C}=\frac{2\langle\mathbf{v} \cdot \mathbf{b}\rangle}{\langle\mathbf{v}\rangle^{2}+\langle\mathbf{b}\rangle^{2}}$,

2) the normalized residual energy $\sigma_{R}$

$\sigma_{R}=\frac{\langle\mathbf{v}\rangle^{2}-\langle\mathbf{b}\rangle^{2}}{\langle\mathbf{v}\rangle^{2}+\langle\mathbf{b}\rangle^{2}}$

where $\mathbf{v}$ and $\mathbf{b}$ are the fluctuating velocity and magnetic field vectors with $\mathbf{b}$ in Alfvén units (i.e. $\mathbf{b} \rightarrow \mathbf{b} / \sqrt{4 \pi \rho}$ where $\rho$ is the plasma density), and $\langle\cdot \cdot\rangle$ denotes time averaging. These parameters can only vary in the range $[-1,1]$, where $\sigma_{C}=+1(-1)$ when only outward (inward) components are present, and $\sigma_{R} \neq 0$ in absence of equipartition between magnetic and kinetic fluctuations. Figure 6 shows the bidimensional histogram in the plane $\sigma_{C}-\sigma_{R}$ at 2-h scale [i.e. the averages in expressions (23) and (24) are evaluated on a

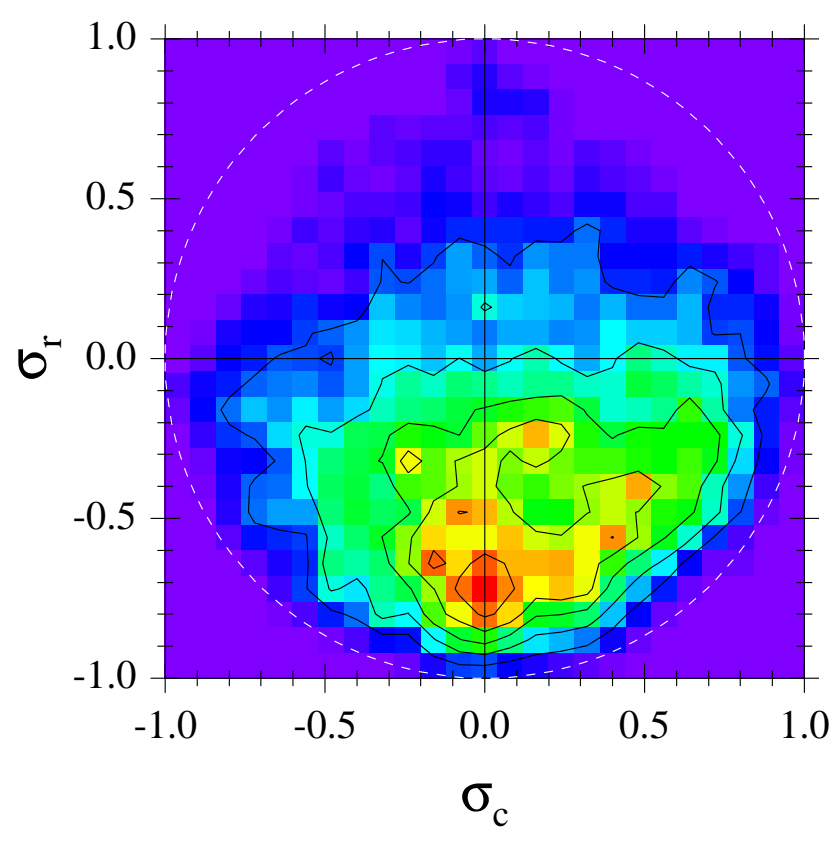

Fig. 6. The distribution of the two parameters $\sigma_{C}$ and $\sigma_{R}$ at the 2-h scale. Colors range from violet to red for increasing frequency values. Bins are $0.08 \times 0.08$ in amplitude. The dashed white circle denotes the locus $\sigma_{C}^{2}+\sigma_{R}^{2}=1$.

2-h scale]. From Fig. 6 we can note that the selected period is mainly dominated by non-Alfvénic fluctuations. As a matter of fact, the maximum occurrence is observed in the range $-0.1 \leq \sigma_{C} \leq 0.1$ and $-0.8 \leq \sigma_{R} \leq-0.6\left(\left\langle\sigma_{C}\right\rangle \sim 0.08\right.$, $\left\langle\sigma_{R}\right\rangle \sim-0.32$ ), suggesting the presence of fluctuations dominated by magnetic field variations with no-velocity fluctations. This result supports the existence of magnetic field structures as already discussed in many previous works (see e.g. Bavassano et al., 1998, 2000), making the selected period a good candidate to test the proposed PDF model.

\subsection{Results}

As said, to evaluate the PDF of the magnetic field intensity and of its small-scale differences (usually named fluctuations) we considered the $24 \mathrm{~s}$ magnetic field intensity averages to remove spurious effects due to satellite spin characteristic time scale. Figure 7 shows the PDF of the magnetic field intensity for the selected period in comparison with a 3-D Maxwell-Boltzmann distribution,

$P\left(x \mid s_{o}\right)=\frac{4 \pi}{\left(2 \pi s_{0}\right)^{\frac{3}{2}}} x^{2} \exp \left(-\frac{x^{2}}{2 s_{0}}\right)$,

where the variance $s_{0}$ is the same of the considered timeseries. Such a PDF has been chosen as a reference for a pure 3-D stochastic Gaussian process. The actual PDF of magnetic field intensity shows systematic deviations form the Maxwell PDF. In particular, the right-hand tail of the 


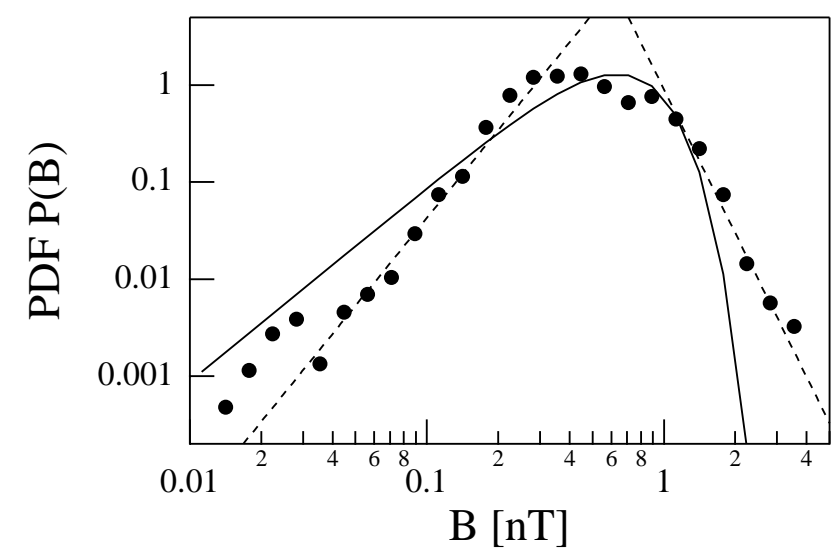

Fig. 7. The PDF $P(B)$ of the magnetic field intensity (solid circles) for the selected period. The solid line refers to a 3-D Maxwell distribution characterized by the same variance of the considered signal. Dashed lines are power-law fits.

actual PDF shows a non-exponential decrease with increasing values of $B$. By fitting the tails of the PDF using a power-law expression $\left(P \sim B^{\eta}\right)$ we get $\eta=[3.01 \pm 0.03]$ and $\eta=[-4.93 \pm 0.07]$ for the left-hand and right-hand tail, respectively. This result is very well in agreement with the predictions of expression (8), and allows us to estimate the product $\alpha \beta$, which is [3.97 \pm 0.05$]$. Furthermore, at very small values some deviations from the power-law behavior are observed $(B<0.03 \mathrm{nT})$. These deviations are associated to a few tens of measurements out of more than $6.4 \cdot 10^{5}$, perhaps related with crossings of regions where the mean field polarity changes.

Knowing the first and second moment of the actual timeseries permits us to estimate the $x_{0}$ and $\beta$ parameters by expressions (9) and (10) simply solving numerically the following expression for fixed $\alpha$,

$\frac{\langle x\rangle^{2}}{\left\langle x^{2}\right\rangle}=\frac{\Gamma^{2}\left(\alpha-\frac{1}{\beta}\right) \Gamma^{2}\left(\alpha+\frac{1}{\beta}\right)}{\Gamma^{2}(\alpha) \Gamma\left(\alpha-\frac{2}{\beta}\right) \Gamma\left(\alpha+\frac{2}{\beta}\right)}$,

and, then, to evaluate $x_{0}$ from one of the two expression (9) and (10). In Table 1 we reported the estimated parameters for different $\alpha$. While, as expected, the parameter $\beta$ shows a dependence on $\alpha$, the characteristic value $x_{0}$ does not change substantially, showing a variability of less than $1 \%$ in the explored parameter range.

On the basis of the values reported in Table 1 and of the value of the product $\alpha \beta$ as estimated by the previous consideration on the tail dependencies, we set for the generalized Weibull parameters the following ones: $\alpha=5 / 3, \beta=2.43$ and $x_{0}=0.641[\mathrm{nT}]$. A comparison between the PDF of the actual magnetic field intensity and the randomized Weibull PDF with the above set of parameters is shown in Fig. 8. The agreement is very good except for very small values of $B$ $(<0.03 \mathrm{nT})$.
Table 1. Estimated parameters of randomized Weibull PDF.

\begin{tabular}{cccc}
\hline$\alpha$ & $\beta$ & $x_{0}$ & $\alpha \beta$ \\
\hline 1 & 3.59 & 0.647 & 3.59 \\
$\frac{4}{3}$ & 2.86 & 0.643 & 3.81 \\
$\frac{3}{2}$ & 2.62 & 0.642 & 3.93 \\
$\frac{5}{3}$ & 2.43 & 0.641 & 4.05 \\
2 & 2.14 & 0.640 & 4.28 \\
\hline
\end{tabular}

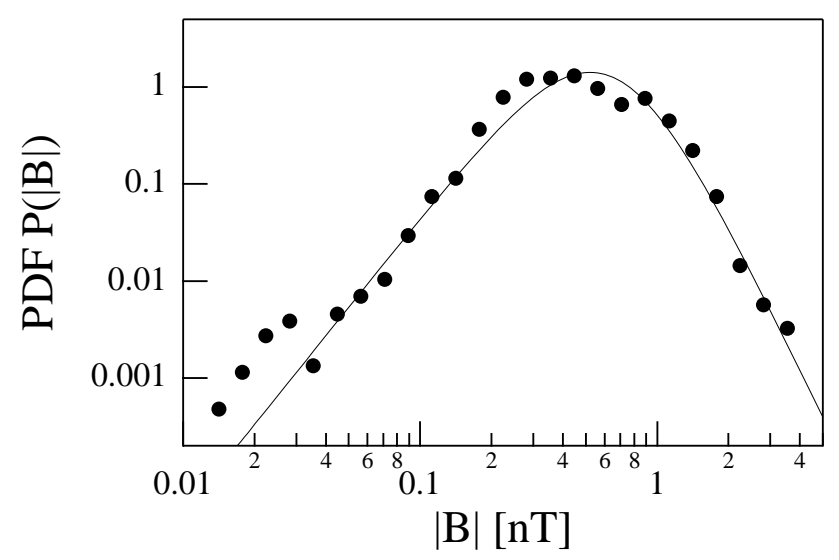

Fig. 8. Comparison between the PDF of the actual magnetic field intensity measurements (solid circles) and the randomized Weibull PDF with $\alpha=\frac{5}{3}, \beta=2.43$ and $x_{0}=0.641$ [nT] (solid line).

To analyze the statistical properties of the small-scale (24 s) magnetic field intensity increments (fluctuations), we computed the small-scale differences:

$\delta B(t)=B(t+\tau)-B(t)$

where $\tau=24 \mathrm{~s}$. We reported in Fig. 9 the PDFs $P(\delta B)$ of the small-scale increments. The shape is clearly nonGaussian and leptokurtotic, with a very pronounced central part and enhanced tails. The characteristic scale associated with the small-scale increments is $\sqrt{\delta B^{2}} \sim 0.014 \mathrm{nT}$, a value more than one order of magnitude less than the one predicted on the basis of the PDF of the magnetic field intensity for a Markov process $\left(\sqrt{\delta B^{2}} \sim x_{0}\right)$. However, the tails at $|\delta B|>0.2 \mathrm{nT}$ scale according to a power-law with an exponent near the one predicted by Eqs. (11) and (8): $P(\delta B) \rightarrow \delta B^{-\mu}$ with $\mu=\alpha \beta+1 \sim-5$. A possible explanation of the difference in the second moments between the PDF of the actual magnetic field differences, and the PDF predicted using the model stands in the non-Markovian feature of the magnetic field intensity time-series. This means that, although the PDF of actual B-values follows expression (8), in the $\{B\}$-time series $B_{i+1}$ is expected to be near the previous $B_{i}$ of the field: this is the signature of a certain degree of persistence in the $\{B\}$-time series. So, if we rearrange the 


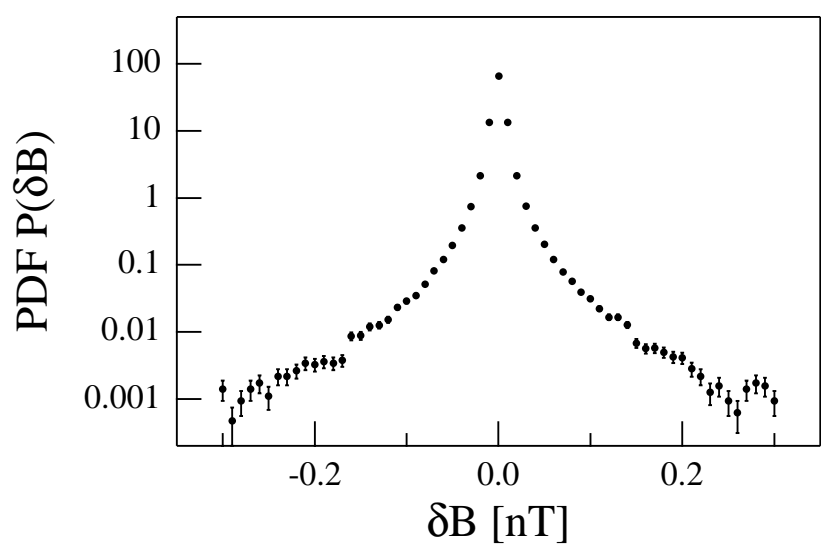

Fig. 9. The PDFs $P(\delta B)$ of the small-scale increments at $\tau=24 \mathrm{~s}$.

actual time-series of magnetic field measurements randomly permuting the time occurrence of the set of observed $\{B\}$ values we will get a PDF of small scale increments approaching the one predicted by the randomized Weibull model.

Figure 10 shows the PDF $P\left(\delta B_{r}\right)$ of the small-scale increments relative to the randomized time-series obtained by permuting the time occurrences $\left(10^{6}\right.$ permutations $)$. As expected, the obtained PDF approaches the one predicted by the randomized Weibull PDF with a $\sqrt{\delta B_{r}^{2}} \sim x_{0}$. This result suggests the existence of persistence in the actual magnetic field intensity time-series, as for instance it occurs in a Lévy flight. In other words, this can be considered an alternative check for the existence of correlation in respect to the usual evaluation of the autocorrelation function. We would like to remark that the observed persistence supports the idea on the existence of magnetic field structures in the solar wind (see e.g. Bruno and Carbone, 2005 and references therein). Here, the word "structure" refers to a certain state ( $B$-value) which perdures in time.

\section{Conclusions}

In this work we have presented a statistical approach, based on rare event statistics and subordination method, to the statistics of magnetic field intensity. The main results of our work can be summarized as follows:

- the observed PDF of the magnetic field intensity is in agreement with the proposed randomized Weibull;

- the PDF of the small-scale differences (24s) of the magnetic field intensity shows a clear discrepancy in terms of characteristic moments from the one predicted by the randomized Weibull distribution assuming the absence of any correlation in the signal.

In the light of the obtained results it is natural to ask ourselves what could be the physical implications and what we

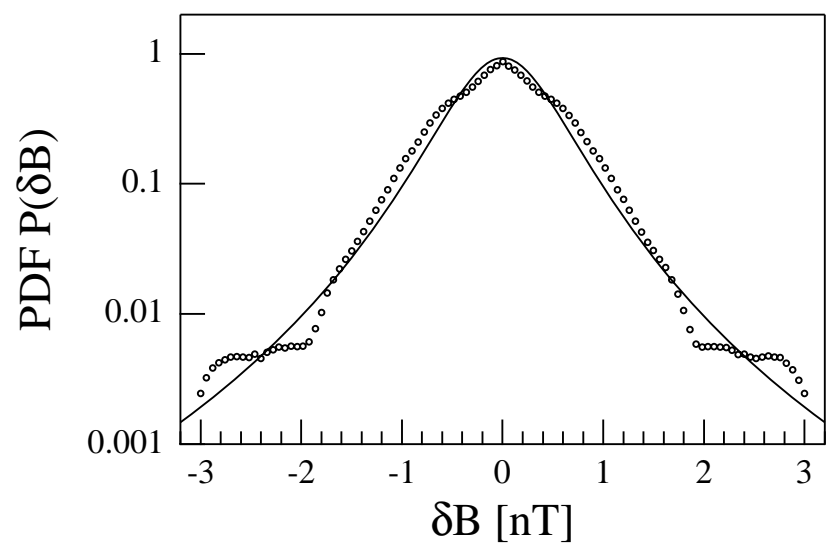

Fig. 10. The PDFs $P\left(\delta B_{r}\right)$ of the small-scale increments of permutated time-series. The solid line is the PDF predicted by the randomized Weibull model.

can learn from the exercise we have presented in this work. To clarify the physical implications it is necessary to come back to the physical meaning of Eq. (5) and to put our attention to the discrepancy between the actual PDF of the magnetic field intensity differences and the one expected by the randomized Weibull model. The randomization procedure of the distribution parameters in Eq. (5) can be read as a way to assume the presence of heterogeneity in the system (time-series) under investigation. On the other hand, the discrepancy between the predicted and the observed PDF of the small-scale differences points toward the presence of correlations in the magnetic field intensity. Combining these two results, we can figure out a scenario consisting of a comprise of heterogeneous multi-scale magnetic field structures in a mechanical local quasi-equilibrium condition (see below). In other words, the agreement between the predicted randomized Weibull and the actual PDF of magnetic field intensity fluctuations indicates that the observed magnetic field is the super-position/aggregation of different families of magnetic field structures.

These conclusions are well in agreement with previous predictions and findings on the existence of flux-tubes and structures in solar wind (Ness et al., 1966; McCracken and Ness, 1966; Mariani et al., 1973; Tu and Marsch, 1990, 1993; Bruno et al., 2001; Borovsky, 2008). On the other hand, 2DMHD simulations by Wu and Chang (2000) (see also Chang et al., 2004) evidenced the formation of field-aligned coarsegrained coherent structures emerging out of a pure stochastic scenario. In this framework the principal merit of our analysis is to have shown the heterogeneity of these structures from the side of magnetic field intensity.

Regarding the existence of a mechanical local quasiequilibrium condition in this flux-tube texture it can be inferred again of the basis of Eq. (5). As a matter of fact, as said in Sect. 2, the directing process is analogous to impose the existence of a sort of equilibrium condition among the 
different components of the system itself (Lavenda, 1995a, b). In our case we consider the magnetic field intensity $B$ which is representative of the compressive part of the solar wind. Thus, Eq. (5) is analogous to assume a sort of magnetic-pressure equilibrium condition in the flux-tube texture, perhaps reflecting the existence of a near mechanical local equilibrium condition. Clearly, this assertion is mainly speculative and has to be confirmed in the framework of a more wide thermodynamic scenario. As a matter of fact, it requires a careful analysis of other thermodynamic parameters (thermal pressure, energy density, entropy, etc.) in the considered period.

At this point one could ask how much general the results of our analysis could be in the wide context of space plasmas. We believe that the proposed scenario could be valid in each region of space where magnetic field fluctuations dominate on Alfvénic fluctuations. For instance, we do not feel that a similar scenario could be valid for high-speed solar wind flowing out from the high-latitude coronal holes, a region where the observed turbulence seems to be more homogeneous and Alfvénic (Bavassano et al., 1998, 2000; Bruno and Carbone, 2005).

Last but not least, it is necessary to stress that the model proposed in this work could be not unique in the choice of the source PDF (here the generalized Weibull distribution). However, we believe that apart from the choice of the initial PDF, following Lavenda (1995a, b) we have clearly shown a way how to introduce heterogeneity in modeling the PDF of space plasma parameters.

Acknowledgements. For the data used in this work we acknowledge the Ulysses Principal Investigators of FGM and SWOOPS, A. Balogh (Imperial College, London, UK) and D. McComas (Southwest Research Institute, USA), M. Lancaster and C. Tranquille of the Ulysses Data System (ESA/ESTEC, NL) and the NSSDC (USA) and NASA-CDAweb (USA) service staffs.

Edited by: B. Tsurutani

Reviewed by: two anonymous referees

\section{References}

Angelopoulos, V., Mukai, T., and Kokubun, S.: Evidence for intermittency in Earth's plasma sheet and implications for selforganized criticality, Phys. Plasmas, 6, 4161-4168, 1999.

Bavassano, B., Pietropaolo, E., and Bruno, R.: Cross-helicity and residual energy in solar wind turbulence - Radial evolution and latitudinal dependence in the region from 1 to $5 \mathrm{AU}$, J. Geophys. Res., 103, 6521, 1998.

Bavassano, B., Pietropaolo, E., and Bruno, R.: Alfvénic turbulence in the polar wind: A statistical study on cross helicity and residual energy variations, J. Geophys. Res., 105, 12697-12704, 2000.

Bavassano, B., Pietropaolo, E., and Bruno, R.: Alfvénic fluctuations in newborn polar solar wind, Ann. Geophys., 23, 1513-1520, 2005 ,

http://www.ann-geophys.net/23/1513/2005/.
Beck, C. and Cohen, E. G. D.: Superstatistics, Physica A, 322, 267275, 2003.

Bertin, E. and Clusel, M.: Generalized extreme value statistics and sum of correlated variables, J. Phys. A, 39, 7607-7619, 2006.

Biskamp, D.: Nonlinear Magnetohydrodynamics, Cambridge university Press, Cambridge, 1993.

Borovsky, J. E.: Flux tube texture of the solar wind: Strands of the magnetic carpet at 1 AU?, J. Geophys. Res., 113, doi: 10.1029/2007JA012684, 2008.

Bruno, R., Carbone, V., Veltri, P. et al.: Identifying intermittency events in the solar wind, Planet. Space, Sci., 49, 1201-1210, 2001.

Bruno, R. and Carbone, V.: The solar wind as a turbulence laboratory, Living Reviews in Solar Physics, 2(4), 2005.

Castaing, B., Gagne, Y., and Hopfinger, E. J.: Velocity probability density functions of high Reynolds number turbulence, Physica D, 46, 177-200, 1990.

Chang, T.: Low-dimensional behavior and symmetry breaking of stochastic systems near criticality - Can these effects be observed in space and in the laboratory?, IEEE T. Plasma. Sci., 20, 691694, 1992.

Chang, T.: Self-organized criticality, multi-fractal spectra, sporadic localized reconnections and intermittent turbulence in the magnetotail, Phys. Plasmas, 6, 4137-4145, 1999.

Chang, T., Tam, S. W. Y., and Wu, C.-C.: Complexity induced anisotropic bimodal intermittent turbulence in space plasmas, Phys. Plasmas, 11, 1287-1299, 2004.

Chapman, S. C., Rowlands, G., and Watkins, N. W.: Extremum statistics: a framework for data analysis, Nonlin. Processes Geophys., 9, 409-418, 2002,

http://www.nonlin-processes-geophys.net/9/409/2002/.

Consolini, G.: On the complexity of the Earth's magnetotail, in: Turbulence and Nonlinear Processes in Astrophysical Plasmas (CP932), edited by: Shaikh, D. and Zank, G. P., AIP, 2007.

Feller, W.: An Introduction to Probability Theory and its Applications vol. II, Wiley, New York, 1971.

Frisch, U.: Turbulence: The Legacy of A. N. Kolmogorov, Cambridge University Press, Cambridge, 1995.

Frisch, U. and Sornette, D.: Extreme deviations and applications, J. Phys. I France, 7, 1155-1171, 1997.

Gumbel, E. J.: Statistics of Extremes, Dover Publications Inc., 2004.

Jung, S. and Swinney, H. L.: Velocity difference statistics in turbulence, Phys. Rev. E, 72, 026304, doi:10.1103/PhysRevE.72.026304, 2005.

Lavenda, B. H.: Thermodynamics of Extremes, Albion Publ., Chichester, 1995a.

Lavenda, B. H.: Subordination and Bayes' theorem in thermodynamics of composite systems, Int. J. Theor. Phys., 34, 615, 1995b.

Leubner, M. P. and Vörös, Z.: A Nonextensive Entropy Approach to Solar Wind Intermittency, Astrophys. J., 618, 547-555, 2005a.

Leubner, M. P. and Vörös, Z.: A nonextensive entropy path to probability distributions in solar wind turbulence, Nonlin. Proc. Geophys., 12, 171-180, 2005b.

Klimas, A. J., Vassiliadis, D. V., Baker, D. N., et al.: The organized nonlinear dynamics of the magnetosphere, J. Geophys. Res., 101, 13089-13114, 1996.

Mariani, F., Bavassano, B., Villante, U., Ness, N. F.: Variations of 
the occurrence rate of discontinuities in the interplanetary magnetic field, J. Geophys. Res., 78(34), 8011-8022, 1973.

Marsch, E. and Tu, C. Y.: Marsch, E. and Tu, C. Y.: Non-Gaussian probability distributions of solar wind fluctuations, Ann. Geophys., 12, 1127-1138, 1994,

http://www.ann-geophys.net/12/1127/1994/.

McCracken, K. G. and Ness, N. F.: The collimation of cosmic rays by interplanetary magnetic field, J. Geophys. Res., 71, 3315, 1966.

Milovanov, A. V. and Zelenyi, L. M.: Milovanov, A. V. and Zelenyi, L. M.: Functional background of the Tsallis entropy: "coarsegrained" systems and "kappa" distribution functions, Nonlin. Processes Geophys., 7, 211-221, 2000,

http://www.nonlin-processes-geophys.net/7/211/2000/.

Ness, N. F., Scearce, C. S., and Cantarano, S.: Preliminary results from the Pioneer 6 magnetic field experiment, J. Geophys. Res., 71, 3305, 1966.

Nicolis, G. and Prigogine, I.: Self-Organization in Nonequilibrium Systems. From Dissipative Structures to Order Through Correlations, Wiley, New York, 1977.

Parisi, G. and Frisch, U.: On the singularity of fully developed turbulence, in: Turbulence and Predictabiliy in Geophysical Fluid Dynamics, Proc. Intern. School of Physics ' E. Fermi , 84-87, edited by: Ghil, M., Benzi, R., and Parisi, G., North-Holland, Amsterdam, 1985.

Sornette, D.: Critical Phenomena in Natural Sciences: Chaos, Fractals, Selforganization and Disorder: Concepts and Tools, Springer, 2003.

Sorriso-Valvo, L., Carbonea, V., Giuliani, P., et al.: Intermittency in plasma turbulence, Planet. Space Sci., 49(12), 1193-1200, 2001.
Tetrault, D.: Turbulent relaxation of magnetic fields, 1. Coarsegrained dissipation and reconnection, J. Geophys. Res., 97, 8531-8540, 1992a.

Tetrault, D.: Turbulent relaxation of magnetic fields, 2. Selforganization and intermittency, J. Geophys. Res., 97, 8541-8547, 1992b.

Tu, C. Y. and Marsch, E.: Evidence for a background spectrum of solar wind turbulence in the inner heliosphere, J. Geophys. Res., 95(A4), 4337-4341, 1990.

Tu, C. Y. and Marsch, E.: A model of solar wind fluctuations with two components: Alfvén waves and convective structures, J. Geophys. Res., 98(A2), 1257-1276, 1993.

Tu, C. Y. and Marsch, E.: MHD structures, waves and turbulence in the solar wind: Observations and theories, Space Sci. Rev., 73, 1-210, 1995.

Vörös, Z., Baumjohann, W., Nakamura, R., Runov, A., Volwerk, M., Schwarzl, H., Balogh, A., and Rème, H.: Dissipation scales in the Earth's plasma sheet estimated from Cluster measurements, Nonlin. Processes Geophys., 12, 725-732, 2005, http://www.nonlin-processes-geophys.net/12/725/2005/.

Weygand, J. M., Kivelson, M. G., Khurana, K. K., et al.: Plasma sheet turbulence observed by Cluster II, J. Geophys. Res., 110, A01205, doi:10.1029/2004JA010581, 2005.

Wu, C.-C. and Chang, T.: 2D MHD simulation of the emergence and merging of coherent structures, Geophys. Res. Lett., 27, 863-866, 2000.

Zelenyi, L. M. and Milovanov, A. V.: Fractal topology and strange kinetics: from percolation theory to problems in cosmic electrodynamics., Phys. Uspekhi, 47, 749-788, 2004. 